\title{
Characterization of genes involved in ceramide metabolism in the Pacific oyster (Crassostrea gigas)
}

\author{
Emma Timmins-Schiffman and Steven Roberts ${ }^{*}$
}

\begin{abstract}
Background: The lipid signaling molecule, ceramide, is a key component of the vertebrate stress response, however, there is limited information concerning its role in invertebrate species. In order to identify genes involved in ceramide metabolism in bivalve molluscs, Pacific oyster genomic resources were examined for genes associated with ceramide metabolism and signaling.

Results: Several genes were identified including full-length sequences characterized for serine palmitoyltransferase-1, 3-ketodihydrosphingosine reductase, acid ceramidase, and ceramide glucosyltransferase. Genes involved in ceramide synthesis and metabolism are conserved across taxa in both form and function. Expression analysis as assessed by quantitative PCR indicated all genes were expressed at high levels in gill tissue. The role of the ceramide pathway genes in the invertebrate stress response was also explored by measuring expression levels in adult oysters exposed to Vibrio vulnificus. Two genes demonstrated increased expression during the bacterial challenge: a gene involved in hydrolytic breakdown of ceramide (acid ceramidase) and a gene involved in de novo generation of ceramide (3-ketodihydrosphingosine reductase), suggesting a possible role of ceramide in the invertebrate stress and immune responses.
\end{abstract}

Conclusions: In silico and laboratory results support that Pacific oysters have the basic components of the ceramide metabolism pathway. These results also indicate that ceramide may have analogous functions in vertebrates and invertebrates. The gene expression pattern of acid ceramidase and 3-kethodihydrosphingosine reductase in response to bacterial exposure especially supports that ceramide and sphingolipid metabolism may be involved in the oyster's stress and/or immune responses.

Keywords: Oyster, Crassostrea gigas, Stress, Ceramide, Bacteria

\section{Background}

Ceramide is a sphingolipid that serves as an important signaling molecule for a variety of cellular processes including differentiation, proliferation, inflammation, and apoptosis [reviewed in 1 and 2]. Different stimuli promote either de novo synthesis of ceramide or its catabolic generation from sphingolipids $[1,2]$. The diversity of processes in which ceramide plays a role as a signaling molecule indicates its importance across a variety of life stages and environmental conditions. For example, the accumulation of ceramide can halt embryonic

\footnotetext{
* Correspondence: Sr320@uw.edu

School of Aquatic and Fishery Sciences, University of Washington, Seattle, WA 98105, USA
}

development [3], inhibit insulin signaling [4], and promote apoptosis during cellular stress [5]. The production of ceramide can be triggered by multiple pathways and is sensitive to exogenous stressors [5,6]. In sea bass (Diecentrarchus labrax), changes in intracellular ceramide levels in gill tissue are associated with abrupt shifts in environmental salinity [7]. Leukemia cells exposed to various exogenous stressors (ionizing radiation, hydrogen peroxide, UV radiation, and heat shock) showed elevated levels of ceramide and increased apoptosis [8].

Ceramide metabolism has also been associated with immune-related processes. Cytokines can trigger sphingomyelin hydrolysis, leading to increased production of ceramide, suggesting that ceramide could propagate cytokine signaling [2]. Ceramide also plays a key role in 
the inflammatory response in Homo sapiens dermal fibroblasts by stimulating interleukin-1 mediated prostaglandin E2 production [9].

While the role of ceramide as a signaling molecule in response to stress has been well studied in mammalian systems, there is little information regarding the function and metabolism of ceramide in invertebrates. The primary goal of this study was to characterize genes associated with ceramide metabolism in the intertidal mollusc, the Pacific oyster (Crassotrea gigas). Using an in silico approach, numerous genes associated with ceramide metabolism were identified and complete coding sequences were isolated for select genes. To provide insight into the functional role of ceramide metabolism in the invertebrate stress response, adult $C$. gigas were exposed to the marine bacterium Vibrio vulnificus and the expression levels of four genes involved in the ceramide pathway were assessed. Given the range of environmental conditions experienced by intertidal species, ceramide signaling could be a key component in the cellular response to these environmental changes.

\section{Results}

A total of 23 sequences associated with ceramide metabolism were identified by analyzing publicly available Crassostrea gigas sequences (Table 1). A majority of the genes are homologous to vertebrate genes involved in de novo synthesis, catabolic generation, or enzymatic breakdown of ceramide (Figure 1). Most sequences were derived from contigs generated by assembling short read sequences (see Additional file 1). Of the 23 sequences, 4 were selected for further characterization based on the percent of putative open reading frame identified. These four genes include serine palmitoyltransferase-1 (Cgsptlc1), 3-ketodihydrosphingosine reductase (Cg-3KDSR), acid ceramidase $(\mathrm{Cg}-\mathrm{AC})$, and ceramide glucosyltransferase $(\mathrm{Cg}$-GlcCer). Based on amino acid alignments, complete nucleotide open reading frames were obtained for Cg-sptlc1 [GenBank: JN315146], Cg-3KDSR [GenBank: JN315143], and $C g-A C$ [GenBank: JN315144]. Cg-GlcCer [GenBank: JN315145] is missing a portion of the 3' end of the nucleotide sequence as determined from alignments with full-length sequences in other species.

Table 1 Genes associated with ceramide metabolism in C. gigas from the in silico search

\begin{tabular}{|c|c|c|c|}
\hline Gene ID & Gene description & Species & e-value \\
\hline $\mathrm{Cg}_{-} 4852$ & Serine palmitoyltrasnferase-1 & Pongo abelii & 0 \\
\hline Cg_877 & Acid ceramidase & Rattus norvegicus & $2 \mathrm{E}-146$ \\
\hline Cg_14141 & 3-ketodihydrosphingosine reductase & Mus musculus & $5 e-46$ \\
\hline Cg_29918 & Ceramide glucosyltransferase & Xenopus tropicalis & $9 e-122$ \\
\hline Cg_21728 & Acid sphingomyelinase & Mus musculus & $5 e-46$ \\
\hline Cg_16356 & Ceramide kinase & Homo sapiens & $1 e-27$ \\
\hline Cg_16726 & Cerebrosidase & Mus musculus & $5 e-139$ \\
\hline Cg_17230 & Neutral ceramidase & Oryza sativa & $5 e-96$ \\
\hline Cg_1560 & Caspase 7 & Homo sapiens & $4 e-10$ \\
\hline Cg_23531 & Caspase 8 & Homo sapiens & $8 e-54$ \\
\hline Cg_252 & TNF receptor-associated factor 2 & Mus musculus & $1 e-53$ \\
\hline Cg_3248 & TNF receptor-associated factor 3 & Mus musculus & $2 e-30$ \\
\hline Cg_31180 & TNF receptor-associated factor 4 & Homo sapiens & $4 e-39$ \\
\hline Cg_6808 & Neutral sphingomyelinase & Caenorhabditis elegans & $4 e-10$ \\
\hline Cg_20643 & Dihydrosphingosine 1-phosphate phosphatase & Schizosaccharomyces pombe & $3 e-9$ \\
\hline Cg_26221 & Sphingosine-1-phosphate phosphatase & Mus musculus & $8 e-20$ \\
\hline Cg_7888 & Sphingosine-1-phosphate lyase & Dictyostelium discoideum & $6 e-9$ \\
\hline HS213433 & Sphingomyelin synthase & Homo sapiens & $1 e-100$ \\
\hline HS185280 & Ceramide synthase & Mus musculus & $5 e-83$ \\
\hline HQ425701 & Inhibitor of apoptosis & Crassostrea gigas & - \\
\hline HQ425703 & Caspase 1 & Crassostrea gigas & - \\
\hline HQ425705 & Caspase 2 & Crassostrea gigas & - \\
\hline HQ425699 & Fas-associated receptor with Death Domain & Crassostrea gigas & - \\
\hline
\end{tabular}

Genes associate with ceramide metabolism were identified in C. gigas by searching of publicly available databases. Sequences assembled from short read archive data and expressed sequence tags were given a Gene ID code that corresponds to the sequence in the Additional file 1. For each of these contiguous sequences, the top BLASTx hit description, corresponding species, and e-value are provided. Two genes that matched to a single EST each in GenBank (sphingomyelin synthase and ceramide synthase) are denoted with their respective GenBank Accession Numbers since they are not represented as a contig of multiple ESTs. An additional four genes [GenBank: HQ425699, HQ425701, HQ425703, and HQ425705] have been previously characterized in C. gigas [20]. 


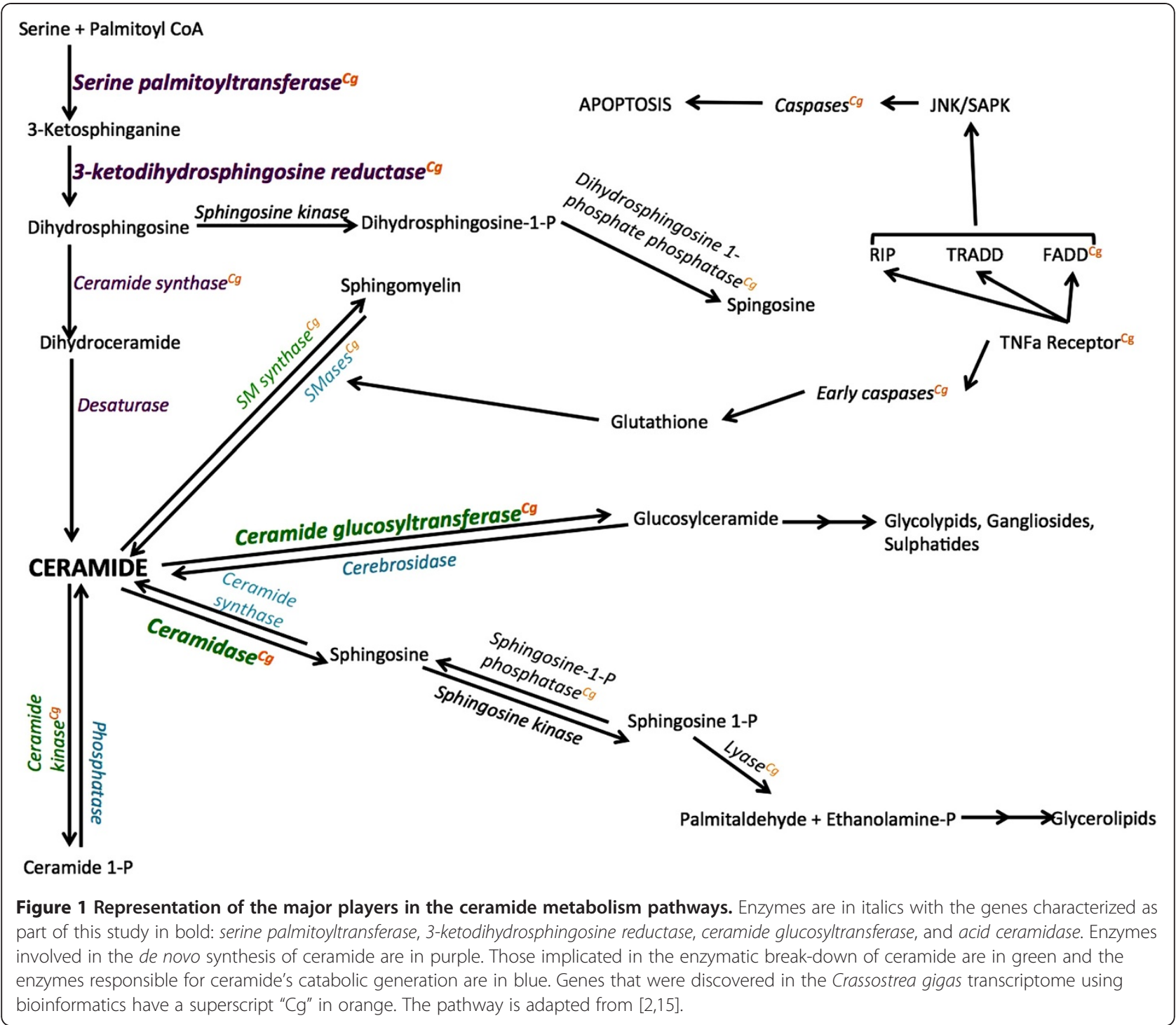

\section{Serine palmitoyltransferase- 1}

The open reading frame of $\mathrm{Cg}$-sptlc1 is $1404 \mathrm{bp}$ and is most similar to sptlc-1 in Xenopus tropicalis and in the hemichordate, Saccoglossus kowalevskii (Table 2). At the amino acid level Cg-sptlc1 is most similar to serine palmitoyltransferase 1 in the Sumatran orangutan, Pongo abelii (Table 2). Based on alignments at the deduced amino acid level, Cg-sptlc1 shares $59.8 \%$ pairwise identity with the $H$. sapiens homolog and $51.0 \%$ pairwise identity over 475 amino acids with Caenorhabditis elegans Sptlc1 (Figure 2).

The highest level of Cg-sptlc1 gene expression was detected in gill tissue, followed by digestive gland, mantle, and then adductor muscle (data not shown). Expression levels in gill tissue were 40 times higher the levels in adductor muscle tissue. Cg-sptlc1 expression was not significantly altered in gill tissue from oysters exposed to
Vibrio (mean expression \pm standard error $=2.4 \mathrm{E}-3 \pm 2.0 \mathrm{E}-4$ ), compared to controls (1.8E-3 $\pm 2.3 \mathrm{E}-4, \mathrm{p}=0.068$; Figure 3). Average gene efficiency calculated in PCR Miner [10] for qPCR was 0.733 (standard deviation $=0.07$ ).

\section{3-ketodihydrosphingosine reductase}

The $C g$ - $3 K D S R$ open reading frame is $1129 \mathrm{bp}$ and is most similar to the Rattus norvegicus 3KDSR sequence and to 3KDSR from Saccoglossus kowalevskii (Table 2). The amino acid translation of $C g$ - $3 K D S R$ is most similar to Mus musculus 3KDSR (Table 2). The Crassostrea gigas amino acid sequence shares $50.8 \%$ identity to the corresponding homolog in H. sapiens (Figure 4). Based on the derived amino acid sequence of Cg-3KDSR, the catalytic site and NADH/NADPH binding site [11] are conserved in oysters (Figure 4). 
Table 2 Top BLASTn and BLASTx hits for the Crassostrea gigas genes Cg-sptlc1, Cg-3KDSR, Cg-GlcCer, Cg-AC

\begin{tabular}{llllcc}
\hline Gene & Top BLASTn Hit & $\begin{array}{l}\text { BLASTn } \\
\text { Similarity }\end{array}$ & $\mathbf{2}^{\text {nd }}$ BLASTn Hit & $\begin{array}{l}\mathbf{2}^{\text {nd }} \text { BLASTn } \\
\text { Similarity }\end{array}$ & Top BLASTx Hit \\
\hline Cg-sptlc1 & Xenopus tropicalis (NM_001079574) & $71 \%$ & $\begin{array}{l}\text { Saccoglossus kowalevskii } \\
\text { (SM_002730516) }\end{array}$ & $70 \%$ & Pongo abelii (Q5R9T5) \\
\hline Cg-3KDSR & Rattus norvegicus (NM_001108342) & $68 \%$ & $\begin{array}{l}\text { Saccoglossus kowalevskii } \\
\text { (SM_002740331) }\end{array}$ & $76 \%$ & Mus musculus (Q6GV12) \\
\hline Cg-GlcCer & $\begin{array}{l}\text { Pediculus humanus corporis } \\
\text { (SM_002431306) }\end{array}$ & $66 \%$ & Xenopus laevis (NM_001090475) & $66 \%$ & Xenopus tropicalis (Q5BL38) \\
\hline Cg-AC & Sebastes schlegelii (AB491143) & $67 \%$ & - & - & Rattus norvegicus (Q6P71) \\
\hline
\end{tabular}

The BLAST algorithm was used to find the best matches in the NCBI nucleotide database (BLASTn) and UniProt/SwissProt protein database (BLASTx). For each gene, the top two BLASTn matches are given along with the percent sequence similarity. Sequence identity values shown represent local similarity as reported by BLAST. Cg-AC only had one top BLASTn hit. The top BLASTx protein hit is also given. GenBank Accession Numbers are in parentheses next to the species name of the top hit.

Gene expression of $C g$-3KDSR was highest in gill tissue with expression levels over 1000 times higher compared to other tissues (data not shown), which had comparable expression of this gene. Cg-3KDSR gene expression in Vibrio-exposed oysters $(8.4 \mathrm{E}-5 \pm 1.1 \mathrm{E}-5)$ was higher than expression in controls (5.8E-5 $\pm 8.5 \mathrm{E}-6, \mathrm{p}=0.037$; Figure 3 ). Average gene efficiency calculated in PCR Miner [10] was 0.918 (standard deviation $=0.08$ ) .

Ceramide glucosyltransferase

Cg-GlcCer (1124 bp) is most similar to ceramide glucosyltransferase from the human body louse, Pediculus humanus corporis, followed by Xenopus laevis UDP-glucose ceramide glucosyltransferase (Table 2). The translated amino acid sequence is most similar to Xenopus tropicalis ceramide glucosyltransferase (Table 2). Crassostrea gigas and $H$. sapiens share a $45.9 \%$ pairwise amino acid identity over 396 residues in the alignment, while C. elegans and C. gigas share $40.9 \%$ pairwise identity over 468 residues (Figure 5).

Cg-GlcCer had a similar expression profile across tissues to Cg-sptlc1, the highest expression being in the gill, followed by digestive gland, mantle and adductor (data not shown). The gene expression data for the bacterial exposure were square-root transformed based on a $\lambda$ of 0.42 . The $\mathrm{Cg}$-GlcCer gene 
was not expressed differently in Vibrio-exposed oysters $(2.4 \mathrm{E}-8 \pm 3.3 \mathrm{E}-9)$ compared to control oysters $(3.8 \mathrm{E}-8 \pm 1.1 \mathrm{E}-8, \mathrm{p}=0.98$; Figure 3$)$. Average gene efficiency calculated in PCR Miner [10] was 0.898 (standard deviation $=0.16)$.

\section{Acid ceramidase}

The open reading frame for $C g-A C$ is $1170 \mathrm{bp}$ in length and was most similar to the gene BRF 7-G7 in Sebastes schlegelii (Table 2). The translated amino acid sequence for $C$. gigas is the most similar to R. norvegicus acid ceramidase (Table 2). The $\mathrm{Cg}-\mathrm{AC}$ amino acid sequence shares $46.6 \%$ pairwise identity over 402 residues in the alignment with $C$. elegans and $49.4 \%$ identity with the $H$. sapiens sequence over 398 residues (Figure 6).

$C g-A C$ was expressed the most in the gill tissue followed by digestive gland, mantle, and adductor (data not shown). The gene expression data for the bacterial exposure were subjected to a reciprocal transformation based on a $\lambda$ of -0.87 . The expression of $C g-A C$ was significantly higher in
Vibrio-exposed oysters $(2.2 \mathrm{E}-3 \pm 2.8 \mathrm{E}-3)$ compared to controls $(1.6 \mathrm{E}-3 \pm 1.3 \mathrm{E}-4, \mathrm{p}=0.038$; Figure 3$)$. Average gene efficiency calculated in PCR Miner [10] was 0.876 (standard deviation $=0.06$ ).

All four genes showed similar phylogenetic topologies (Figure 7), with the amino acid sequences clustering into distinct invertebrate and vertebrate lineages. When the $C$. elegans sequence was available and included in the phylogeny, it clustered with the $C$. gigas sequence with a bootstrap value of $100 \%$. H. sapiens and M. musculus sequences always clustered together with a bootstrap of $100 \%$.

\section{Discussion}

Gene identification

This study identified a suite of genes in the Pacific oyster homologous to vertebrate genes associated with ceramide metabolism, including the sequencing and characterization of serine palmitoyltransferase-1 (Cg-sptlc1), acid ceramidase $(C g-A C)$, 3-ketodihydrosphingosine reductase (Cg-3KDSR), 
and ceramide glucosyltransferase ( $\mathrm{Cg}$-GlcCer). These data provide an important resource for further studies that focus on the role of ceramide in the environmental response in invertebrates. While well studied in vertebrate systems, there have been only a few recent studies that focus on ceramide metabolism and signaling in molluscs [see 12-14].

Numerous genes associated with ceramide metabolism are conserved across distant taxonomic lineages. In vertebrates, the genes described here are directly responsible for synthesis of ceramide (sptlc1, 3KDSR; Figure 1) and generation of sphingolipids from ceramide (AC and GlcCer). In silico analysis of the Crassostrea gigas transcriptome shows that there are a number of other genes in these ceramide metabolism pathways (Table 1). In fact, almost all the genes coding for enzymes necessary for de novo ceramide synthesis were identified, suggesting a conservation of this metabolic pathway in C. gigas. The only gene not found was that coding for the enzyme desaturase that converts dihydroceramide to ceramide (Figure 1). However, further research into the presence, structure, and functional role of ceramide is needed to confirm these suggestive results. Additionally, a number of enzymes responsible for transformation of ceramide into other lipid products were identified including ceramide kinase, ceramide synthase, and sphingomyelin synthase. A variety of caspases, TNF superfamily receptors, RIP (receptor-interacting serine/threonine-protein) and FADD (Fas-associated protein with death domain) subunits of the TNF $\alpha$ (tumor necrosis factor) receptor, which are key components of the cellular stress and apoptotic responses, were also identified in public databases. These receptors are implicated up- and downstream of ceramide signaling pathways in vertebrates $[2,15]$. Several genes known to be involved in ceramide metabolism were not found in this effort (i.e. dihydroceramide desaturase, ceramide- $1 \mathrm{P}$ phosphatase). This is likely related to the fact that these genes have yet to be sequenced in the Pacific oyster. It is also possible that corresponding enzymes lack significant sequence homology. Once a complete genome is sequenced for this species, a more comprehensive analysis can be performed.

\section{Gene characterization}

Sptlc1, responsible for accumulation of intracellular ceramide during cellular stress [5,16], is highly conserved in oysters including a 21 residue transmembrane region 


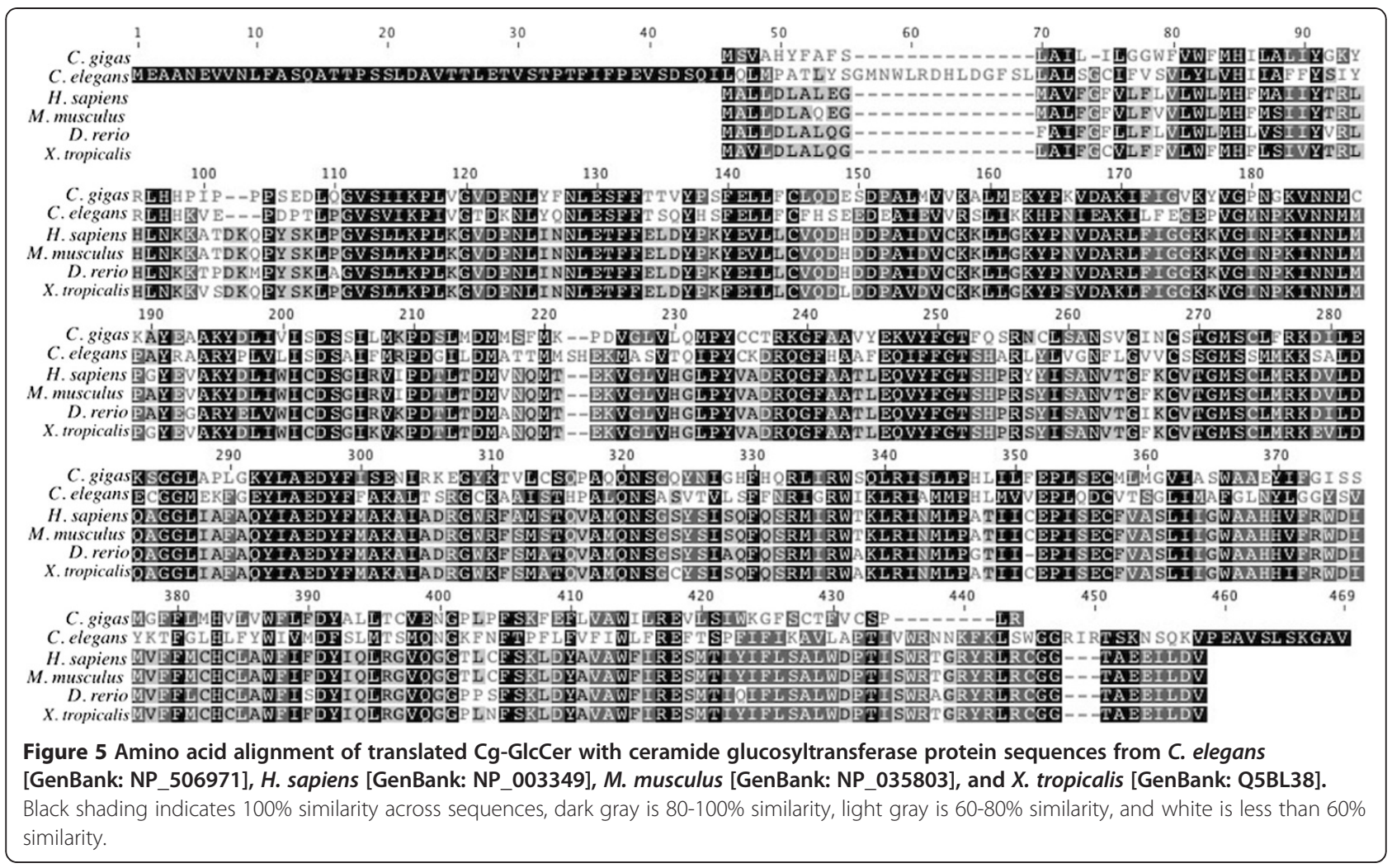

originally identified in $H$. sapiens [17]. The serine palmitoyltransferase identified in $C$. gigas has high homology with the LCB1 $\mathrm{H}$. sapiens isoform. There are two forms of
H. sapiens Sptlc - LCB1 and LCB2 [17]. Homo sapiens LCB2 has a conserved motif that binds pyridoxal phosphate $[17,18]$, but LCB1 has an asparagine instead, which

\section{\begin{tabular}{|llllllll}
\hline 1 & 10 & 20 & 30 & 40 & 50 & 60 & 70
\end{tabular}

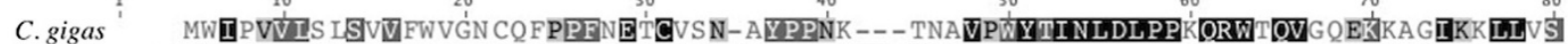

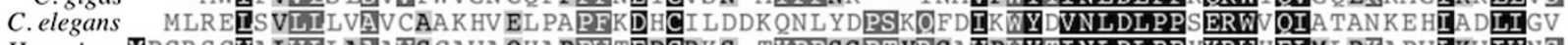

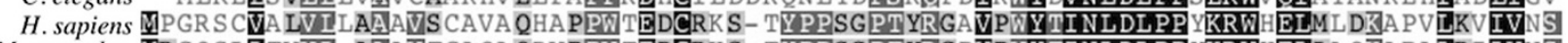

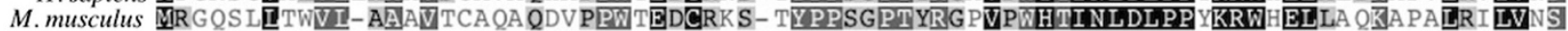

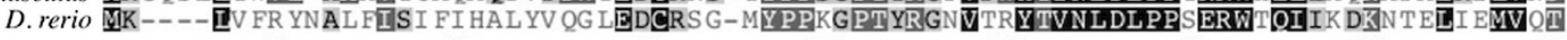

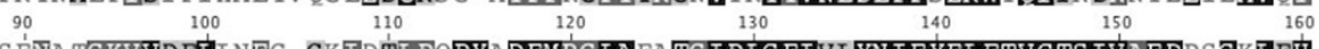

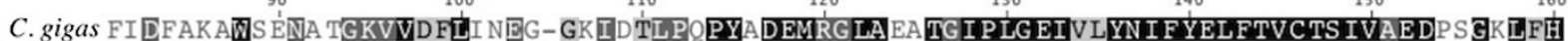

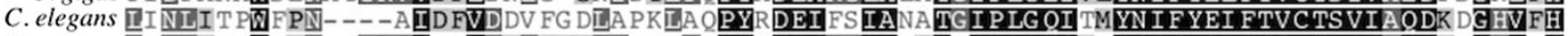

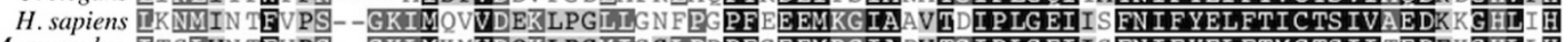

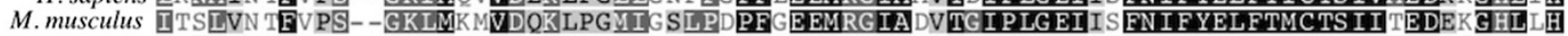 \\ D. rerio IK DMAK GE FH---GKLVN FVDKELPFIVD TLPN PENEEI KGIAAVSGI PLGEI ALFN IFYEVFTVCTS LVAEDNNGNIY田

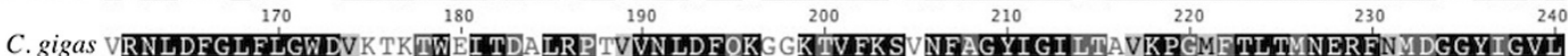

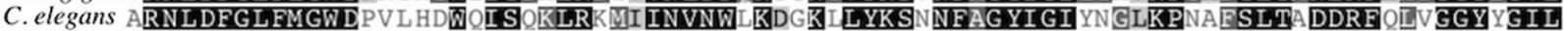

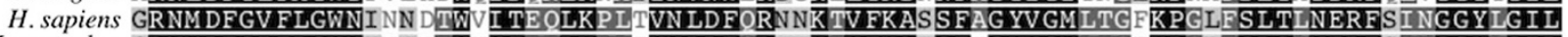

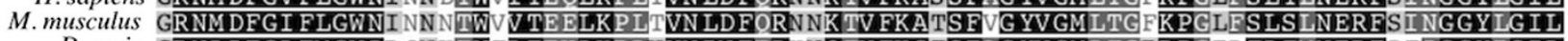 \\ D. rerio GRNLDFGLFMGW DR ONKTWTLTEKLKPLVVN IN FERK NONVFKSTSFAG YVGMLTGI RPGELTLTMNERFDF DGGYIGIL

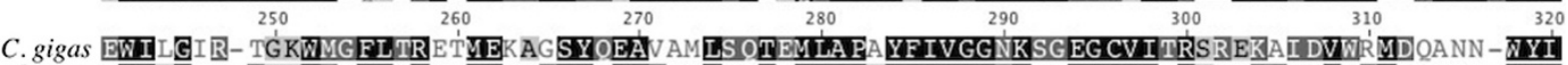

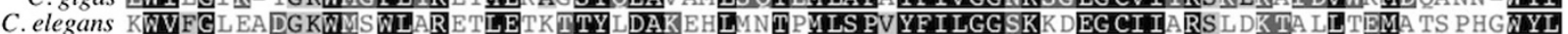

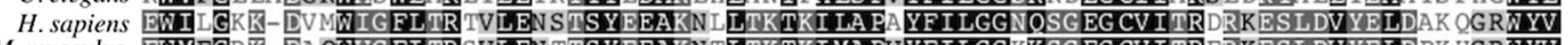 M. musculus EWMFGR

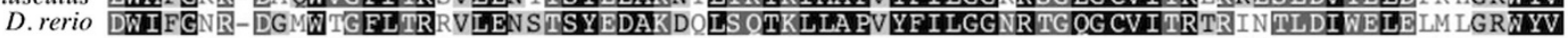

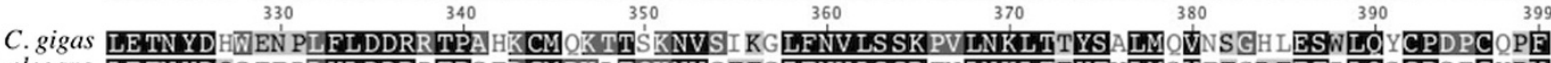 \\ C. elegans LETNYDOG TEDLYLDDRDTPG FRCMDKLTOKNVGFE IFNV LSSRTN LNKLTTYTVLMSUTESR FETI LOS CPG ECYPW

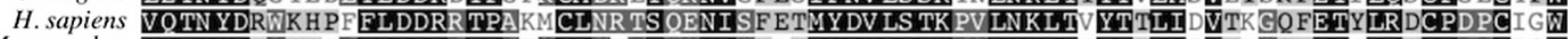

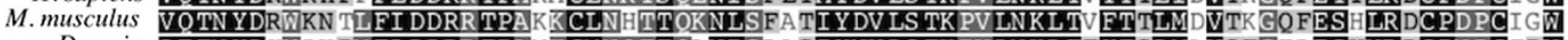

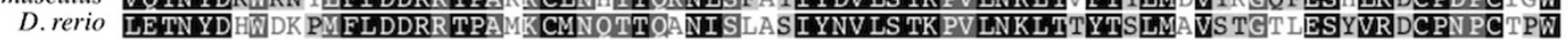

Figure 6 Amino acid alignment of translated Cg-AC with protein sequences from acid ceramidase in C. elegans [GenBank: NP_493173], H. sapiens [GenBank: NP_808592], M. musculus [GenBank: NP_062708], and D. rerio [GenBank: NP_001006088]. Black shading indicates

$100 \%$ similarity across sequences, dark gray is $80-100 \%$ similarity, light gray is $60-80 \%$ similarity, and white is less than $60 \%$ similarity. 


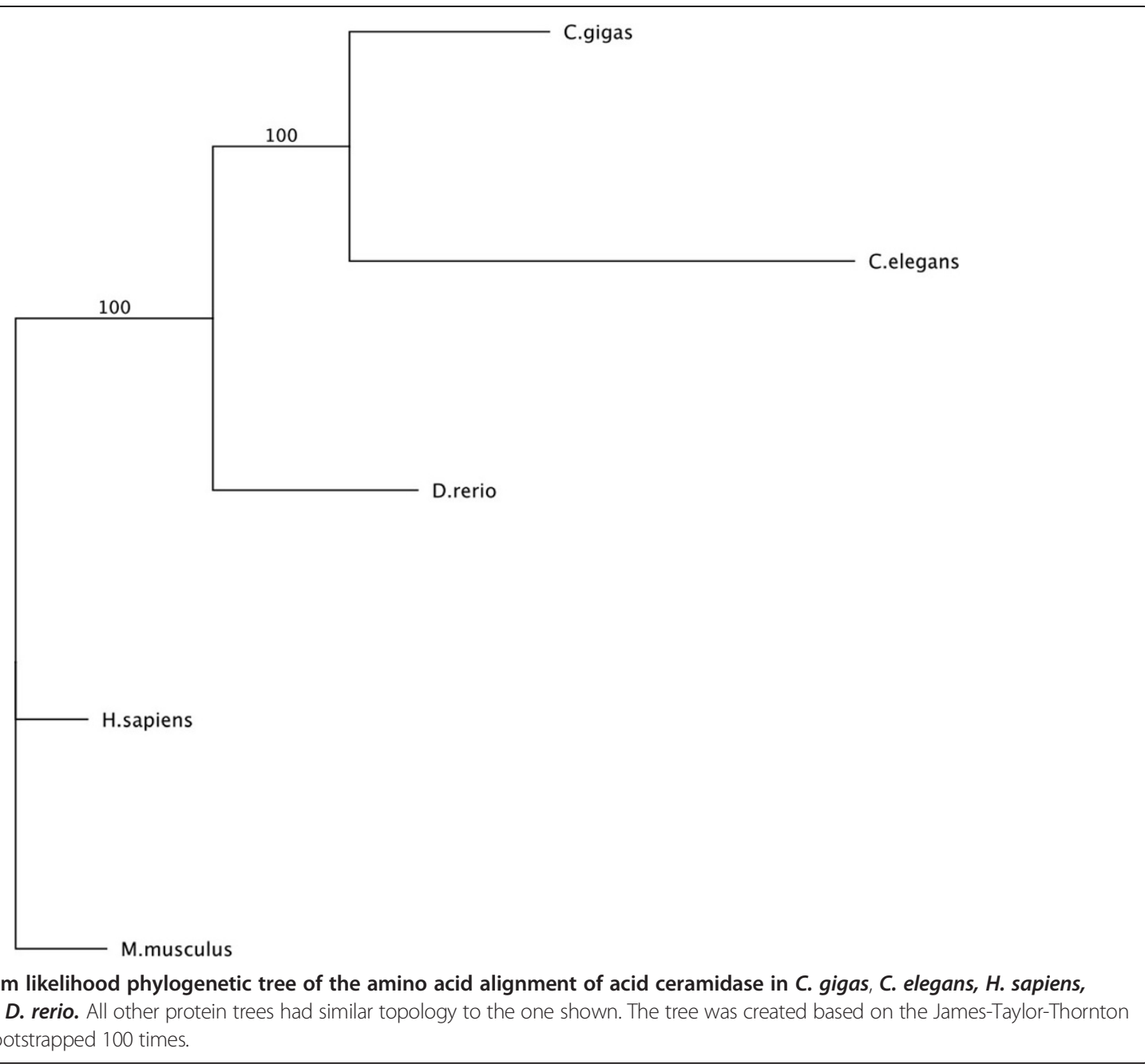

is homologous to the C. gigas sequence. In Homo sapiens, LCB1 is necessary for the maintenance of LCB2 and does not perform the same catalytic functions [17]. More research is needed to determine if the functionality of specific Sptlc isoforms are conserved across taxa.

Crassostrea gigas 3KDSR shares conserved catalytic domains with all other amino acid sequences in the alignment, suggesting that its functionality is conserved across taxonomic groups. 3-ketodihydrosphingosine reductase acts downstream of Sptlc. The product of the reaction catalyzed by Sptlc is 3-ketosphinganine. 3-ketosphinganine is reduced by a NADPH-dependent reductase to dihydrosphingosine. The enzyme that catalyzes this reaction is 3-ketodihydrosphingonsine reductase (3KDSR). 3KDSR contains two functional sites that are highly conserved, based on the amino acid alignment: an NADH/NADPH binding site and a catalytic site [11] (Figure 4). All four amino acid sequences $-C$. gigas, $H$. sapiens, $M$. musculus, and $D$. rerio - share more of the catalytic site motif than previously described: Tyr-Ser-X-Ser-Lys, beginning at position 187 on the alignment [11] (Figure 4). The motif of the NADH/NADPH binding site is identical in its entirety - Gly-Gly-Ser-Ser-Gly-Ile-Gly - across all four sequences/ taxa [11] (Figure 4).

\section{Implications for the stress response}

The gene expression patterns observed for each gene suggest a role of ceramide metabolism in the oyster stress response. The highest expression was observed in the gill tissue, which is rich with hemocytes, the primary immune cell in bivalves. Furthermore, exposure to Vibrio vulnificus significantly elevated expression of $C g-A C$ and $C g-3 K D S R$. There are several possible interpretations of this increase in gene expression that corroborate the second messenger role of ceramide in oysters. The increased expression of both enzymes suggests that the bacterial exposure prompted up-regulation of ceramide production (effected by $C g$-3KDSR) followed by its metabolism into other sphingolipids (by $C g-A C$ ). There are a number of reasons why ceramide may be transformed into other lipid 
products during environmental stress. For instance, increased expression of $C g-A C$ could indicate that ceramide is transformed into the lipid sphingosine, which then functions downstream as a signaling molecule in the C. gigas immune response. Sphingosine is an important signaling molecule in the vertebrate immune response and probably plays a similar role in invertebrates. Sphingosine is associated with the inhibition of the proliferation of Th2 T cells, inhibition of protein kinase $\mathrm{C}$ activity, regulation of the complement system, and inhibition of neutrophil respiratory burst $[19,20]$. Bivalves possess a complex immune system that has many homologous components to the vertebrate immune system [21], thus it is probable that secondary signaling molecules like ceramide are instrumental in immune response coordination. The roles that sphingosine and other sphingolipids play in the immune response seem to be heavily influenced by their concentrations [20], thus $C g-A C$ could be a pivotal enzyme regulating levels of sphingosine in oyster, at least in a short-term response.

An alternative explanation for the increased expression of $C g-A C$ during Vibrio vulnifcus challenge suggests that ceramide is the primary signaling molecule in the $C$. gigas immune response. An accumulation of ceramide in response to the $V$. vulnificus exposure could have occurred and $\mathrm{Cg}$ $A C$ may be up-regulated to metabolize ceramide after it has performed its signaling roles. Ceramide may have been produced to increase signaling of immune pathways necessary for responding to bacterial exposure. Increased expression of $\mathrm{AC}$ has been shown to decrease intracellular ceramide in mammals $[4,6]$ and could very well play the same role in invertebrates. The relatively greater expression of $\mathrm{Cg}$ $3 K D S R$ in Vibrio-exposed oysters supports this second hypothesis.

\section{Conclusions}

Here we report the identification of numerous genes in Crassostrea gigas that are homologous to genes involved in vertebrate metabolism of ceramide, an important lipid signaling molecule. Gene expression analysis suggests that ceramide is involved in the immune response of oysters exposed to microbial pathogens. It should be noted that a limited number of genes were examined here and targeted studies would be required to further elucidate the functional role of ceramide metabolism in bivalves. For instance, future efforts might directly quantify sphingolipid levels and correlate levels with specific cellular function or use of fluorescence in situ hybridization to show cellular gene expression. Assays of gene expression are sometimes merely suggestive of a true, functional organismal response effected by changes in protein expression. Changes in global gene expression, however, are indicative of a significant physiological response, at least at the cellular level, and frequently these changes are correlated with changes in protein expression [22]. It is not known if lipid content in bivalve diets impacts stress physiology by influencing ceramide levels. Le Grand et al. [23] discovered that ceramide-based phosphophingolipids are an important component of Pacific oyster hemocyte membranes. Characterizing how diet and other conditions affect ceramide metabolism could offer a framework for better understanding mechanisms associated with environmental effects on immune function.

\section{Methods}

\section{Gene discovery}

Genes involved in Crassostrea gigas ceramide metabolism were identified using publicly available sequence data. Specifically, short read sequences from $C$. gigas larvae complementary DNA (cDNA) libraries [GenBank: SRX032364] as well as all expressed sequence tags (ESTs) were downloaded from NCBI (www.ncbi.nlm.nih.gov). All sequences were quality trimmed and de novo assembled using CLC Genomics Workbench v3.7 (CLC bio, Katrinebjerg, Denmark). Consensus sequences from short read and EST assemblies were compared to the UniProtKB/Swiss-Prot database (http://www.uniprot.org) using NCBI's BLASTx algorithm [24]. Sequences having a top blast hit with an e-value less that $1 \mathrm{E}-5$ were inspected for genes associated with ceramide metabolism. Only genes with an e-value less than $1 \mathrm{E}-30$ were considered for sequencing and gene expression characterization in this study. Individual sequence alignments were performed to determine percent coverage and sequence similarity (Geneious Pro v. 4.8.5 [25]). Four genes were chosen for sequencing and quantitative PCR (qPCR) analysis: serine palmitoyltransferase-1, 3-ketodihydrosphingosine reductase, acid ceramidase, and ceramide glucosyltransferase.

\section{Bacterial challenges}

Adult $C$. gigas (mean length $=9.0 \mathrm{~cm}$, range of 7.9$10.3 \mathrm{~cm}$ with standard deviation of $0.58 \mathrm{~cm}$ ) were obtained from Taylor Shellfish Farms, Inc. (Quilcene, WA) and acclimated to lab conditions for a few weeks. For bacterial challenges, Vibrio vulnificus was grown in $400 \mathrm{~mL}$ culture medium (1x standard Luria-Bertani broth with an additional $1 \% \mathrm{NaCl}$ ) at $37^{\circ} \mathrm{C}$ for 18 hours at $150 \mathrm{rpm}$. The culture was then centrifuged for $10 \mathrm{~min}$ utes at $4300 \mathrm{rpm}\left(25^{\circ} \mathrm{C}\right)$, the supernatant was removed and the pelleted bacteria were resuspended in $50 \mathrm{~mL}$ non-sterile of seawater. Eight oysters held in $8 \mathrm{~L}$ of aerated seawater were inoculated with $V$. vulnificus at an initial concentration of $4.56 \times 10^{18} \mathrm{CFU} / \mathrm{L}$ via a 3 hour immersion bath. Control oysters $(n=8)$ were likewise placed in $8 \mathrm{~L}$ of aerated seawater. Following exposure, oysters were harvested aseptically and gill tissue was dissected and immediately frozen at $-80^{\circ} \mathrm{C}$. Tissues from a single, non-exposed oyster (gill, mantle, adductor 
muscle, and digestive gland) were dissected using sterile techniques and stored in RNAlater (Ambion, Carlsbad, CA) for use in a qPCR assay of differential gene expression across tissues. RNA isolation, reverse transcription and quantitative PCR analysis were carried out as described below.

\section{Gene sequencing}

RNA isolation was carried out using Tri-Reagent (Molecular Research Center, Cincinnati, $\mathrm{OH}$ ) per the manufacturer's protocol. Following RNA isolation, samples were treated with the Turbo DNA-free Kit, rigorous protocol (Ambion) to remove potential genomic DNA carry-over. All samples were evaluated to ensure genomic DNA was absent by performing quantitative polymerase chain reaction (qPCR) on DNAsed RNA samples. Quality and quantity of RNA were determined using a NanoDrop ND-1000 Spectrophotometer (Thermo Fisher Scientific, Waltham, MA). Equal quantities of DNased RNA samples were reverse transcribed using M-MLV reverse transcriptase according to manufacturer's protocol (Promega, Madison, WI).

For genes where the putative open reading frame could be determined based on sequence alignments, PCR primers were designed to amplify entire coding regions (Primer 3 in Geneious Pro v. 4.8.5; [25,26]) (Table 3). Equal quantities of within-treatment gill cDNA were pooled for sequencing PCR reactions. PCR reactions $(25 \mu \mathrm{l})$ were carried out with $12.5 \mu \mathrm{L} 2 \mathrm{x}$ Apex RED Taq Master Mix (Genesee Scientific, San Diego, CA), $8.5 \mu \mathrm{L}$ nuclease-free water, $0.5 \mu \mathrm{L}$ of $10 \mu \mathrm{M}$ forward and reverse primers (Integrated DNA Technologies, Coralville, IA), and $3 \mu \mathrm{L}$ cDNA template. Thermal cycling parameters were as follows: $95^{\circ} \mathrm{C}$ for 10 minutes; 40 cycles of: $95^{\circ} \mathrm{C}$ for 30 seconds, $55^{\circ} \mathrm{C}$ for 30 seconds, and $72^{\circ} \mathrm{C}$ for 30 seconds; $72^{\circ} \mathrm{C}$ for 10 minutes. PCR products were separated on agarose gels, checked for expected amplicon size, excised, cloned in pCR 2.1-TOPO Vector, and transformed in to One Shot Top10 chemically competent cells using the TOPO TA Cloning Kit (Invitrogen, San Diego, CA). Plasmid DNA was isolated from bacterial cultures using the Qiagen MiniPrep Kit, following the manufacturer's protocol (Qiagen, Valencia, CA) and sequenced at the High Throughput Genomics Unit (University of Washington) on an Applied Biosystem's 3730xl (Carlsbad, CA) using vector-specific primers (Invitrogen).

\section{Protein phylogeny}

Sequences were trimmed to their open reading frame and translated to their amino acid sequences (Geneious Pro v. 4.8.5 [25]). Sequence alignments were performed using ClustalX v. 2.1 [27]. Sequences for corresponding proteins in Homo sapiens, Mus musculus, Danio rerio, Xenopus tropicalis and Caenorhabditis elegans were downloaded from NCBI's Homologene database where available. Using the PhyML plugin in Geneious [25,28], maximum likelihood phylogenetic trees of the protein sequences were constructed based on the James-Taylor-Thornton (JTT) model and bootstrapped 100 times [28,29].

\section{Quantitative PCR}

Expression levels across tissue types $(n=1)$ and in response to bacterial exposure ( $\mathrm{n}=8$ per treatment) of all four genes and of the housekeeping gene, elongation factor $1 \alpha(E F 1 \alpha)$, were quantified using qPCR. DNA-free RNA was reverse transcribed to cDNA as described above. qPCR was performed using $1 \mu \mathrm{L}$ of $\mathrm{cDNA}$ diluted 1:20 in nuclease-free water in a $25 \mu \mathrm{L}$ reaction containing $12.5 \mu \mathrm{L}$ of $2 \mathrm{x}$ Immomix Master Mix (Bioline, London, UK), $0.5 \mu \mathrm{L}$ of $10 \mu \mathrm{M}$ forward and reverse primers, $1.0 \mu \mathrm{L} 50 \mu \mathrm{M}$ SYTO13 (Invitrogen), and $9.5 \mu \mathrm{L}$ nuclease-free water. Primers used for qPCR are listed in Table 3. Thermal cycling and fluorescence detection were performed using a CFX96 Real-Time Detection System (Bio-Rad, Hercules, CA). Cycling parameters were as follows: $95^{\circ} \mathrm{C}$ for 10 minutes; 40 cycles of $95^{\circ} \mathrm{C}$ for 15 seconds, $55^{\circ} \mathrm{C}$ for 15 seconds, $72^{\circ} \mathrm{C}$ for $30 \mathrm{sec}-$ onds. Immediately after cycling, a melting curve protocol was run to verify that a single product was generated in each reaction. Duplicate runs for each gene were always run on the same plate to avoid inter-run variability.

Table 3 Sequencing and qPCR primers for ceramide metabolism genes

\begin{tabular}{|c|c|c|c|c|c|}
\hline Gene description & $\begin{array}{c}\text { Elongation } \\
\text { factor } 1 \text { a (EF1 } a)\end{array}$ & $\begin{array}{c}\text { Serine } \\
\text { palmitoyltransferase } \\
\text { (Sptlc1) }\end{array}$ & $\begin{array}{l}\text { 3-ketodihydrosphingosine } \\
\text { reductase (3KDSR) }\end{array}$ & $\begin{array}{l}\text { Acid ceramidase } \\
\text { (AC) }\end{array}$ & $\begin{array}{l}\text { Glucosylceramide } \\
\text { synthase (GlcCer) }\end{array}$ \\
\hline Sequencing Forward Primer & - & $\begin{array}{c}\text { ATGGCGTCGACGTTCA } \\
\text { TTCC }\end{array}$ & AGCGACGGACCGAACTTACT & $\begin{array}{l}\text { TGTGATTACACGAT } \\
\text { GTGGATACCG }\end{array}$ & $\begin{array}{c}\text { AGAGGCGAACACA } \\
\text { CGAAAGT }\end{array}$ \\
\hline Sequencing Reverse Primer & - & $\begin{array}{c}\text { CTGTTCCCCAATATTTC } \\
\text { TGAC }\end{array}$ & TGTCTTGGGTTTTGCATCCTTC & $\begin{array}{c}\text { CTGCTTCTGACTTC } \\
\text { CGGTGT }\end{array}$ & $\begin{array}{l}\text { CCATATGGATAACAC } \\
\text { TTCTCG }\end{array}$ \\
\hline Sequence Product Size (bp) & - & 1483 & 1210 & 1165 & 1080 \\
\hline qPCR Forward Primer & $\begin{array}{c}\text { AAGGAAGCTGCTGA } \\
\text { GATGGG }\end{array}$ & $\begin{array}{l}\text { TTCACAGCAAGCT } \\
\text { GAGCGAT }\end{array}$ & GCAGTGCAGTGGCTGGAAAT & $\begin{array}{l}\text { TGGACTCAAGTTGG } \\
\text { CCAGGA }\end{array}$ & $\begin{array}{c}\text { TTGGCCCAAACGGG } \\
\text { AAAGTT }\end{array}$ \\
\hline qPCR Reverse Primer & $\begin{array}{l}\text { CAGCACAGTCAG } \\
\text { CCTGTGAAGT }\end{array}$ & $\begin{array}{l}\text { AAGTAGCGAGCC } \\
\text { AACGTCAC }\end{array}$ & AGGCAGCCTTGGTGACATTG & $\begin{array}{c}\text { AAGGCTGGGGGAG } \\
\text { AGTATCG }\end{array}$ & $\begin{array}{c}\text { TGTCCATGAGCGAGT } \\
\text { CTGGT }\end{array}$ \\
\hline qPCR Product Size (bp) & 200 & 178 & 168 & 157 & 114 \\
\hline
\end{tabular}


Average Ct (fluorescence-based cycle threshold) values across replicates and average gene efficiencies were calculated with PCR Miner (http://www.miner.ewindup. info/version2 [10]). Gene expression $\left(R_{0}\right)$ was calculated based on the equation $R_{0}=1 /(1+E)^{C t}$, where $E$ is the average gene efficiency and $\mathrm{Ct}$ is the cycle threshold for fluorescence. Each primer pair amplified a single product, as demonstrated by a single melting curve (see Additional file 2). All expression values were normalized to

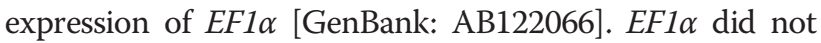
show differential expression between treatments as verified by a $t$-test done on expression values of the qPCR run in duplicate. All qPCRs were run in duplicate and significant differences in expression were determined using a linear model with $\alpha=0.05$. Box-Cox plots were used to assess skewness of gene expression data and determine if transformations needed to be made. The need to transform the data and the transformation used were determined from the lamda $(\lambda)$ corresponding to the maximum log likelihood in the Box-Cox plot. If $\lambda=1$ fell within the $95 \%$ confidence interval of the maximum log likelihood, then no transformation was used. All statistical analyses were done in $\mathrm{R}$ [30].

\section{Availability of supporting data}

The data set supporting the results of this article is included within the article (and its additional files).

\section{Additional files}

Additional file 1: Contigs of ESTs corresponding to ceramide metabolism pathway genes identified in C. gigas. Contigs are listed by Gene ID and described in Table 1.

Additional file 2: Melting curves for qPCR done on the $\mathbf{4}$ genes. Panel $A$ is the melting curve for $C g-3 K D S R$, panel $B$ is for $C g-A C$, panel $C$ is for $\mathrm{Cg}$-GlcCer, and panel D is for Cg-sptlc1.

\section{Competing interests}

The authors declare that they have no competing interests.

\section{Authors' contributions}

ETS participated in the design of the study, carried out all of the molecular lab work and data analysis, and helped to draft the manuscript. SR helped in the design of the study in and in drafting and revising the manuscript. Both authors read and approved the final manuscript.

\section{Acknowledgements}

We thank Sam White for his help with the lab work that was part of this study. We would also like to thank Taylor Shellfish, Inc. for their generous donation of oysters. Loveday Conquest provided invaluable assistance with the statistical analysis. Thank you to Mackenzie Gavery, Lisa Crosson, Dave Metzger, and Sam White for their critical review of this manuscript. This research was funded in part by the National Oceanographic and Atmospheric Administrations Saltonstall-Kennedy Grant and in part by the University of Washington's School of Aquatic and Fishery Sciences. All the authors of this paper have read the manuscript and have agreed to have their names listed as authors. All those listed as having contributed critical review of this paper have also agreed to have their names included in the acknowledgements section.
Received: 1 June 2012 Accepted: 6 September 2012

Published: 13 September 2012

\section{References}

1. Hannun YA: The sphingomyelin cycle and the second messenger function of ceramide. J Biochem Chem 1994, 269:3125-3128.

2. Ballou LR, Laulederkind SJ, Rosloniec EF, Raghow R: Ceramide signaling and the immune response. Biochim Biophys Acta 1996, 1301:273-287.

3. Eliyahu E, Park J-H, Shtraizent N, He X, Schuchman EH: Acid ceramidase is a novel factor required for early embryo survival. FASEB J 2007, 21:1403-1409.

4. Chavez JA, Holland WL, Bär J, Sandhoff K, Summers SA: Acid ceramidase overexpression prevents the inhibitory effects of saturated fatty acids on insulin signaling. J Biol Chem 2005, 280:20148-20153.

5. Perry DK, Bielawska A, Hannun YA: Quantitative determination of ceramide using diglyceride kinase. Methods Enzymol 2000, 312:22-31.

6. Strelow A, Bernardo K, Adam-Klages S, Linke T, Sandhoff K, Krönke M, Adam $D$ : Overexpression of acid ceramidase protects from tumor necrosis factor-induced cell death. J Exp Med 2000, 192:601-612.

7. El Babili M, Brichon G, Zwingelstein G: Sphingomyelin metabolism is linked to salt transport in the gills of euryhaline fish. Chem Mater Sci 1996, 31:385-392.

8. Verheij M, Bose R, Lin XH, Yao B, Jarvis WD, Grant S, Birrer MF, Szabo E, Zon LI, Kyriakis JM, Haimovitz-Friedman A, Fuks Z, Kolesnick RN: Requirement for ceramide-initiated SAPK/JNK signaling in stress-induced apoptosis. Lett Nat 1996, 380:75-79.

9. Ballou LR, Chao CP, Holness MA, Barker SC, Raghow R: Interleukin-1mediated PGE2 production and sphingomyelin metabolism. Evidence for the regulation of cyclooxygenase gene expression by sphingosine and ceramide. J Biol Chem 1992, 267:20044-20050.

10. Zhao S, Fernald RD: Comprehensive algorithm for quantitative real-time polymerase chain reaction. Comput Biol 2005, 12:1045-1062.

11. Kihara A, Igarashi Y: FVT-1 is a mammalian 3-Ketodihydrosphingosine Reductase with an active site that faces the cytosolic side of the endoplasmic reticulum membrane. J Biol Chem 2004, 279:49243-49250

12. Lee $Y$, De Zoysa M, Whang I, Lee S, Kim Y, Oh C, Choi CY, S-Y Y, Lee J: Molluscan death effector domain (DED)-containing caspase-8 gene from disk abalone (Haliotis discus discus): Molecular characterization and expression analysis. Fish Shellfish Immunol 2011, 30:480-487.

13. Zhang L, Li L, Zhang G: Gene discovery, comparative analysis and expression profile reveal the complexity of the Crassostrea gigas apoptosis system. Dev Comp Immunol 2011, 35:603-610.

14. Romero A, Estévez-Calvar N, Dios S, Figueras A, Novoa B: New insights into the apoptotic process in mollusks: characterization of caspase genes in Mytilus galloprovincialis. PLoS One 2011, 6:e17003. doi:10.1371/journal. pone.0017003.

15. Hannun YA, Luberto C: Ceramide in the eukaryotic stress response. Trends Cell Biol 2000, 10:73-80.

16. Perry DK: Serine palmitoyltransferase: role in apoptotic de novo ceramide synthesis and other stress responses. Biochimica et Biophysica Acta - Molecular and Cell Biology of. Lipids 2002, 1585:146-152.

17. Hanada K: Serine palmitoyltransferase, a key enzyme of sphingolipid metabolism. Biochimica et Biophysica Acta - Molecular and Cell Biology of. Lipids 2003, 1632:16-30.

18. Momany C, Ghosh R, Hackert ML: Structural motifs for pyridoxal-5'phosphate binding in decarboxylases: An analysis based on the crystal structure of the Lactobacillus 30a ornithine decarboxylase. Protein Sci 2008, 4:849-854

19. Merrill AH Jr, Stevens VL: Modulation of protein kinase $C$ and diverse cell functions by sphingosine - a pharmacologically interesting compound linking sphingolipids and signal transduction. Biochim Biophys Acta 1989, 1010:131-139.

20. Baumruker T, Prieschl EE: Sphingolipids and the regulation of the immune response. Semin Immunol 2002, 14:57-63.

21. Philipp EER, Kraemer L, Melzner F, Poustka AJ, Thieme S, Findeisen U, Schreiber S, Rosenstiel P: Massively Parallel RNA Sequencing Identifies a Complex Immune Gene Repertoire in the lophotrocozoan Mytilus edulis. PLoS One 2012, 7:e33091. doi:10.1371/journal.pone.033091.

22. Lackner DH, Schmidt MW, Wu S, Wolfe DA, Bahler J: Regulation of transcriptome, translation, and proteome in response to environmental stress in fission yeast. Genome Biol 2012, 13:R25. doi:10.1186/gb-2012-13-4-r25. 
23. Le Grand F, Kraffe E, Marty Y, Donaghy L, Soudant P: Membrane phospholipid composition of hemocytes in the Pacific oyster Crassostrea gigas and the Manila clam Ruditapes philippinarum. Comparative Biochemistry and Physiology - Part A: Molecular and Integrative. Physiology 2011, 159:383-391.

24. Altschul SF, Madden TL, Schaeffer AA, Zhang J, Zhang Z, Miller W, Lipman DJ: Gapped BLAST and PSI-BLAST: a new generation of protein database search programs. Nucleic Acids Res 1997, 25:3389-3402.

25. Drummond AJ, Ashton B, Buxton S, Cheung M, Cooper A, Heled J, Kearse M, Moire R, Stones-Havas S, Sturrock S, Thierer T, Wilson A: Geneious v4.5.6. 2010. Available from http://www.geneious.com.

26. Rozen S, Skaletsky HJ: Primer3 on the WWW for general users and for biologist programmers. In Bioinformatics Methods and Protocols: Methods in Molecular Biology. Edited by Drawetz S, Misener S. Totowa, NJ: Humana Press; 2000:365-386.

27. Larkin MA, Blackshields G, Brown NP, Chenna R, McGettigan PA, McWilliam $H$, Valentin F, Wallace IM, Wilm A, Lopez R, Thompson JD, Gibson TJ, Higgins DF: Clustal W and Clustal X version 2.0. Bioinformatics 2007, 23:2947-2948.

28. Guindon S, Gascuel O: A simple, fast, and accurate algorithm to estimate large phylogenies by maximum likelihood. Syst Biol 2003, 52:696-704.

29. Jones DT, Taylor WR, Thornton JM: The rapid generation of mutation data matrices from protein sequences. Comput Appl Biosci 1992, 8:275-282.

30. R Development Core Team: $R$ : A language and environment for statistical computing. Vienna, Austria: R Foundation for Statistical Computing; 2011. www.R-project.org

doi:10.1186/1756-0500-5-502

Cite this article as: Timmins-Schiffman and Roberts: Characterization of genes involved in ceramide metabolism in the Pacific oyster (Crassostrea gigas). BMC Research Notes 2012 5:502.

\section{Submit your next manuscript to BioMed Central and take full advantage of:}

- Convenient online submission

- Thorough peer review

- No space constraints or color figure charges

- Immediate publication on acceptance

- Inclusion in PubMed, CAS, Scopus and Google Scholar

- Research which is freely available for redistribution

Submit your manuscript at www.biomedcentral.com/submit
() Biomed Central 